\title{
Change detection assessment using fuzzy sets and remotely sensed data: an application of topographic map revision
}

\author{
Graciela Metternicht * \\ School of Spatial Sciences, Curtin University of Technology, GPO Box U 1987, Perth 6845, Western Australia, Australia
}

Received 30 September 1998; accepted 29 April 1999

\begin{abstract}
This paper explores a methodology for computing the amount of changes that have occurred within an area by using remotely sensed technologies and fuzzy modelling. The discussion concentrates on the formulation of a standard procedure that, using the concept of fuzzy sets and fuzzy logic, can define the likelihood of changes detected from remotely sensed data. Furthermore, an example of how fuzzy visualisation of areas undergoing changes can be incorporated into a decision support system for prioritisation of areas requiring topographic map revision and updating is presented. By adapting the membership function of the fuzzy model to fit the shape of the histogram characterising the change image (derived from any of the common pre-classification methods of change detection), areas can be identified according to their likelihood of having undergone change during the period of observation. (c) 1999 Elsevier Science B.V. All rights reserved.
\end{abstract}

Keywords: change detection; fuzzy sets; remote sensing; fuzzy logic; map revision

\section{Traditional methods of determining the amount of changes within an area}

Traditional methods of change detection using either air- or satellite-borne remotely sensed data can be broadly divided in two categories: pre-classification and post-classification. Jensen (1997) states that post-classification comparison of changes is the most commonly used method for quantitative analysis. It requires a complete classification of the individual dates of remotely sensed data, whereupon the operator produces a matrix of change that identifies 'from-to' land cover change classes. The main

\footnotetext{
*E-mail: metternicht@vesta.curtin.edu.au
}

drawback with this method is errors in the individual data classification map will also be present in the final change detection.

On the other hand, pre-classification methods detect changes due to variations in the brightness values of the images being compared. Applying any of these approaches, the operator can identify areas of changes, but is unable to label the kind of change. The following pre-classification approaches are commonly used.

- Image difference: The output of image difference indicates that values close to the mean represent areas of 'no change' and magnitudes close to \pm 255 depicts areas of change.

- Image ratio: In ratioing, two geo-referenced or co-registered images from different dates, with one or more bands in an image, are ratioed band by band 
and the data compared on a pixel-by-pixel basis (Singh, 1989).

- Selective principal component (PC) analysis: In temporal change detection using $\mathrm{PC}$ analysis, both the surface proportion and the magnitude of the changed area in an image determine which principal component images will contain change information. It is the relative amount of variance between the changed area and the unchanged part in an image that determines which particular PCs contain change information (Parra et al., 1996).

Another approach commonly adopted by topographic organisations or other individuals interested in change detection is that of manual, on-screen digitising of changes, also known as mono-orthocompilation (or monoplotting). This approach requires enhancement (e.g., by histogram stretching, colour composites and edge enhancement filters) or transformation (e.g., merging SPOT panchromatic and multispectral images, band ratios, Tasselled Cap transformation and edge detection filters) of the more recent image. The enhanced image is then used as a backdrop display for digitising changes on-screen. Modifications are detected by visual inspection or 'searching' of areas of change. To reduce the search space, the operator assumes that new changes generally occur near existing developments. By head-up digitising, features are extracted, labelled and incorporated into a geographic information system (GIS) database. Changes are, therefore, detected in a hybrid GIS by superimposing the existing (vector) database on the recent (raster) image.

\section{The problem of accurately separating areas of change/no change}

In any of the pre-classification approaches, the critical step relies on selecting appropriate threshold values in the lower and upper tails of the histogram representing values of change. This is so that areas of change can be accurately separated from those where no changes have occurred within the period of time considered. In all studies that create a change image, the value at which the threshold is set is somewhat arbitrary. With respect to image difference, Jensen (1996) states that 'most analysts prefer to experiment empirically... the amount of change is often subjective and mainly based on familiarity with the study area...'. Overall, this technique is tedious and time-consuming. Additionally, two types of errors, namely omission and commission can occur when determining these thresholds. Assuming that two separate thresholds for negative and positive

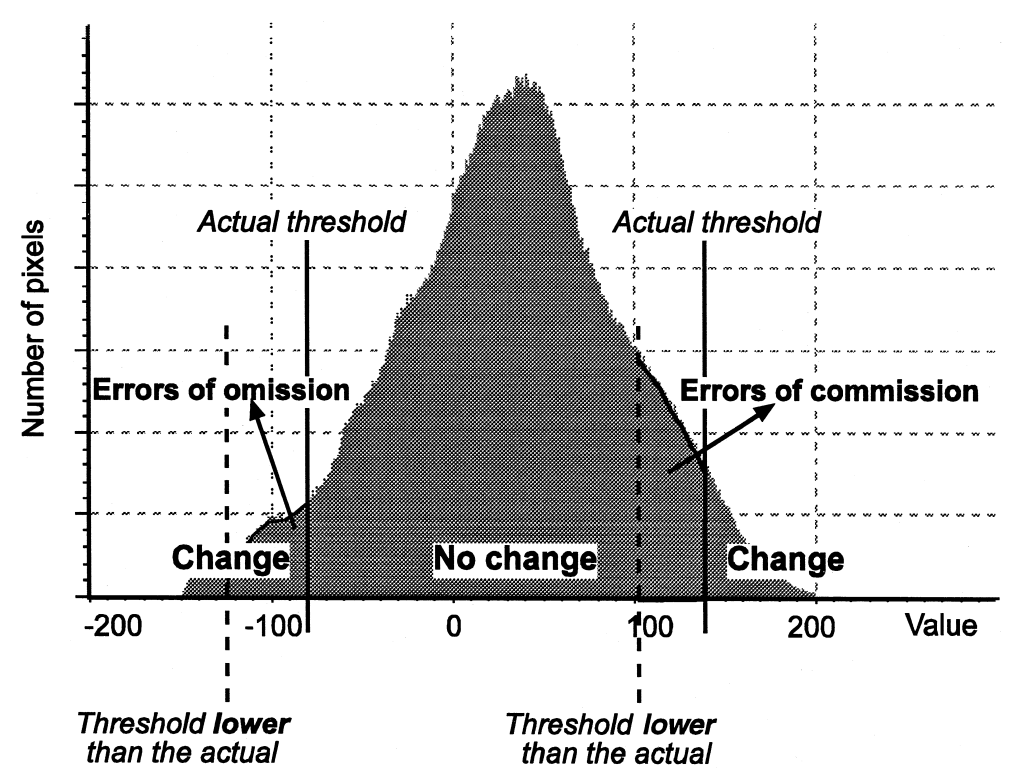

Fig. 1. Graphical representation of the errors of omission and commission. 
differences are used, errors of commission in the estimation of areas that have changed occur when a threshold lower than the actual one for the positive differences is determined, thus including unchanged pixels into the areas of change (Fig. 1). Similarly, errors of omission are produced when the operator sets a threshold lower than the actual one for the negative differences and includes pixels of change in the 'no change' areas.

This status quo calls for a move towards a standard and accurate method for thresholding the 'change images' resulting from the application of any of the above mentioned pre-classification methods. The fuzzy change model presented in this article is among one of the possible responses to this problem. The hypothesis is that by adapting the membership function of the fuzzy model to fit the shape of the histogram characterising the change image, accurate separation of areas of change from those that did not experience change can be assured.

\section{Topographic map revision and change detec- tion}

Map revision operations for existing topographic databases fall into two parts: (a) change detection encompassing feature extraction and classification, and (b) change insertion into the database, integrating the old and new data. The methods of change detection depend, among other things, on the basic mapping scale. Countries having large-scale base maps often use field inspection methods for identifying changes (e.g., the Ordnance Survey, UK), while countries with medium-scale base maps use aerial photographs or satellite imagery (e.g., Germany, Canada, Brazil, Israel and Australia). In his final report as Chairman of the ISPRS working Group IV/3: 'Map and Database Revision', Paul Newby formulated a general review on the state of the art in map and database revision (Newby, 1996). In the course of these discussions, Newby highlights how practices may develop over the coming years under the influence of possible technical changes such as high resolution digital imagery from commercial satellites such as EarthWatch, Space Imaging, Orbview, SPOT 5 and airborne digital cameras.

Regardless of the method of change detection, however, most of the national topographic mapping organisations are faced with the problem of maintaining an updated topographic database in a cost-effective manner. Relying on a procedure to preevaluate the amount of changes that have occurred in an area could help reduce the revision costs and prioritise the areas to be revised and updated in the digital database. This is becoming more relevant, as the current tendency is to sub-contract companies from the private sector to undertake the tasks of digital map revision, feature extraction and their incorporation into the digital topographic database, although the quality control of these tasks still falls under the responsibility of topographic organisations. However, one main problem is estimating the amount of changes that have occurred within an area so that, for instance, a fair quotation in the price to be paid for the execution of these tasks can be fixed prior to the commencement of the work.

Accordingly, this paper explores a methodology for computing land cover changes by using remotely sensed technologies and fuzzy modelling. The discussion concentrates on the formulation of a standard procedure that uses the concept of fuzzy sets and fuzzy logic to define, in an appropriate manner, the likelihood of changes detected from remotely sensed data. Furthermore, an example of how fuzzy visualisation of areas undergoing changes can be incorporated into a decision support system for the prioritisation of areas requiring topographic map revision and updating is outlined.

The approach adopted here is based on early suggestions brought about by Jensen and Toll (1982) and Jensen (1997). They mention that change between dates may not always be classified into discrete classes, but rather, there may exist a continuum of change as a parcel of land could change, for instance, from rangeland to a fully developed residential housing. Thus, it is their recommendation that change detection algorithms should incorporate some fuzzy logic that takes into account the imprecise nature of digital remote sensing change detection. The fuzzy change model presented hereafter intends to fulfil these earlier statements.

\section{Fuzzy sets and fuzzy reasoning}

The concept of fuzzy sets, as defined by Zadeh (1965) is that of a 'class' with a continuum of grades 
of membership. As mentioned by Burrough (1989), it is appropriate to use fuzzy sets whenever we have to deal with ambiguity (e.g., 'What is the threshold value accurately separating areas of change from no-change?'), vagueness and ambivalence in mathematical or conceptual models.

Fuzzy reasoning is based on fuzzy logic, which uses graded or qualified statements rather than ones that are strictly true or false. The results of fuzzy reasoning are not as definite as those derived by strict logic, but they cover a larger field of discourse. For instance, when using fuzzy reasoning it is valid to express that a specific area is "extremely likely to have changed within the period of time being considered', as opposed to the crisp reasoning of change or no-change. Fuzzy logic furnishes a systematic basis for the computation of certainty factors in the form of fuzzy numbers. The numbers may be expressed as linguistic probabilities or fuzzy quantifiers, as for instance, 'likely', 'very unlikely', 'almost certain' or 'extremely likely'. In this perspective, Zadeh (1984) mentions that fuzzy logic has the potential of being an effective tool for the management of uncertainty in expert systems.

\subsection{Defining fuzzy sets}

A fuzzy set can be defined mathematically as follows: let $X$ be a space of points (e.g., pixels), with a generic element of $X$ denoted by $x$, thus $X=\{x\}$.

$A=\left\{x, \mu_{A}(x)\right\} ; x \in X$

A fuzzy set (class) $A$ in $X$ is characterised by a membership function $f_{A}(x)$ which associates with each point in $X$ a real number in the interval $[0,1]$. The value of $f_{A}(x)$ represents the grade of membership of $x$ in $A$ (Zadeh, 1965). This grade, which is not a probability measure but an admitted possibility, corresponds to the degree to which that point is compatible with the concept represented by the fuzzy set. Thus, points may belong to the fuzzy set to a greater or lesser degree as indicated by a larger or smaller membership grade. In other words, the grade of membership is a measure of the compatibility of an object with the concept represented by a fuzzy set.

\subsection{Defining the membership functions}

The membership function of a fuzzy set, usually expressed as $f_{A}(x)$, defines how the grade of membership of $x$ in $A$ is determined. There are two possible ways of deriving these membership functions. The first approach, named by Robinson (1988) as the Similarity Relation Model, resembles cluster analysis and numerical taxonomy in that the value of the membership function is a function of the classifier used. A common version of this model is the fuzzy $k$-means or $c$-means method, used for soil grouping, remote sensing image classification for cloud cover, vegetation analysis, etc., by Wang (1990) and McBratney and de Gruijter (1992). The second approach, known as the Semantic Import Model, uses an a priori membership function with which individuals can be assigned a membership grade. This model is useful in situations where users have a good, qualitative idea of how to group data, but for different reasons are constrained from using the exact associations of the standard Boolean model (Burrough, 1989). This second approach has been adopted in this research.

Several suitable functions, which can be easily adapted to specific requirements, can be used for defining flexible membership grades. A bell-shaped model as described by Dombi (1990) is adopted in this research, where the membership function is written as:

$$
\begin{aligned}
\mu A(x)= & {\left[(1-\nu)^{\lambda-1}(x-a)^{\lambda}\right] } \\
& /\left[(1-\nu)^{\lambda-1}(x-a)^{\lambda}\right. \\
& \left.+\nu^{\lambda-1}(b-x)^{\lambda}\right] ; x \in[a, b] \\
\mu A(x)= & {\left[(1-\nu)^{\lambda-1}(c-x)^{\lambda}\right] } \\
& /\left[(1-\nu)^{\lambda-1}(c-x)^{\lambda}\right. \\
+ & \left.\nu^{\lambda-1}(x-b)^{\lambda}\right] ; x \in[b, c]
\end{aligned}
$$

where: $\lambda$ (sharpness) is an indicator of increasing membership to a fuzzy set (e.g., 'no changes'); $\nu$ (inflection) is the turning point of the function, which can be interpreted as an expectation level; $a$ and $c$ are the typical points of the function, with a membership degree of zero to the fuzzy set considered; and 
(a)

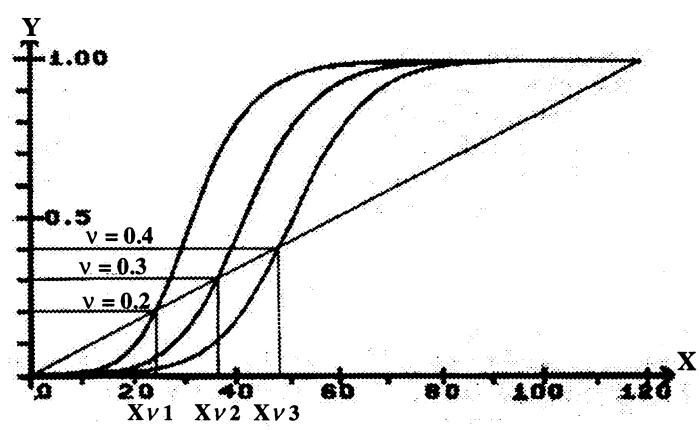

Sharpness $(\lambda)=4$ and $v=0.2,0.3$ and 0.4 (b)

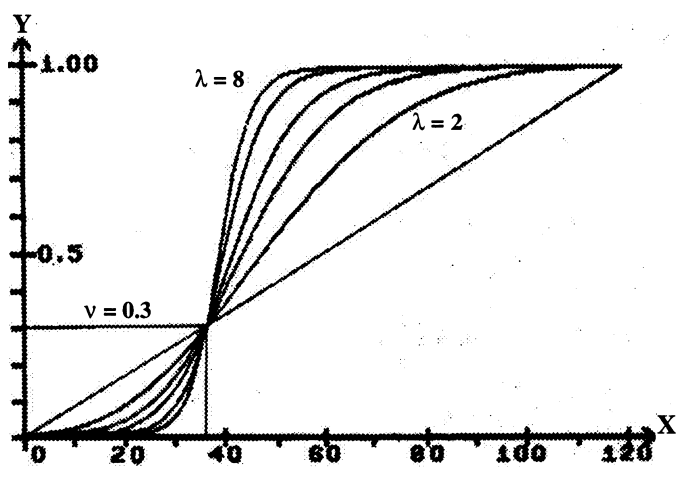

Inflection $(v)=0.3$ and $\lambda=2,3,4,6$ and 8

Fig. 2. Examples of changes in sharpness $(\lambda)$ and inflection $(\nu)$ in the membership function (from Dombi, 1990).

$b$ represents the standard (or ideal) point of the variable $x$ (e.g., the reflectance value characterising areas of no change) at the central concept, that is a grade of membership equal to 1 .

Eqs. (2) and (3) represent the monotonically increasing and decreasing parts of the membership function, respectively. Sharpness and inflection are the two parameters governing the shape of the function. By varying these values, the form of the membership function and the position of the crossover point can be easily controlled (Fig. 2). It is worth mentioning that no rigorous analysis is currently available for choosing the sharpness and inflection of the membership function, although McBratney and Moore (1985) suggest an ad hoc procedure. This research adopts an approach where the sharpness and inflection values are manipulated in such a way that the resulting membership function is in accordance with the shape of the histogram characterising the 'change image'. In this way, it is thought to minimise inaccuracies in the separation of areas of change/no change, produced by histograms of asymmetrical shape.

\section{Implementation of the fuzzy change model}

\subsection{The methodology and data set}

Fig. 3 depicts the methodological approach devised for this research. A set of multi-temporal aerial photographs acquired January 13, 1992 at a scale of 1:40,000 and January 8, 1996 at 1:20,000 scale were available from a previous study sponsored by the Department of Land Administration of Western Australia focused on the feasibility of using digital satellite imagery for map revision tasks at medium scales (Fig. 4) (Metternicht et al., 1997). Changes in the area are related to deforestation and urban development with the construction of new buildings, roads, roundabouts, landscaping and differences in vegetation density in the urban fringe of the Perth City.

One important aspect for accurate change detection is a proper geometric correction in order to minimise the incorporation of false changes due to lack of spatial coincidence between the images being compared. Accordingly, the images were geo-referenced to an existing digital vector database. Twentythree well-distributed control points were selected from the images and their coordinates extracted from the database existing at the Department of Land Administration. First-order polynomial transformation and nearest neighbour resampling techniques were adopted, resulting in root-mean square error (RMSE) values less than the circular map accuracy specified for the mapping scale considered (Metternicht et al., 1997). All data were resampled to $1 \mathrm{~m}$ resolution. Table 1 summarises the characteristics of the data set, number of ground control points, pixel resolution and RMSE values resulting from the geometric correction. 


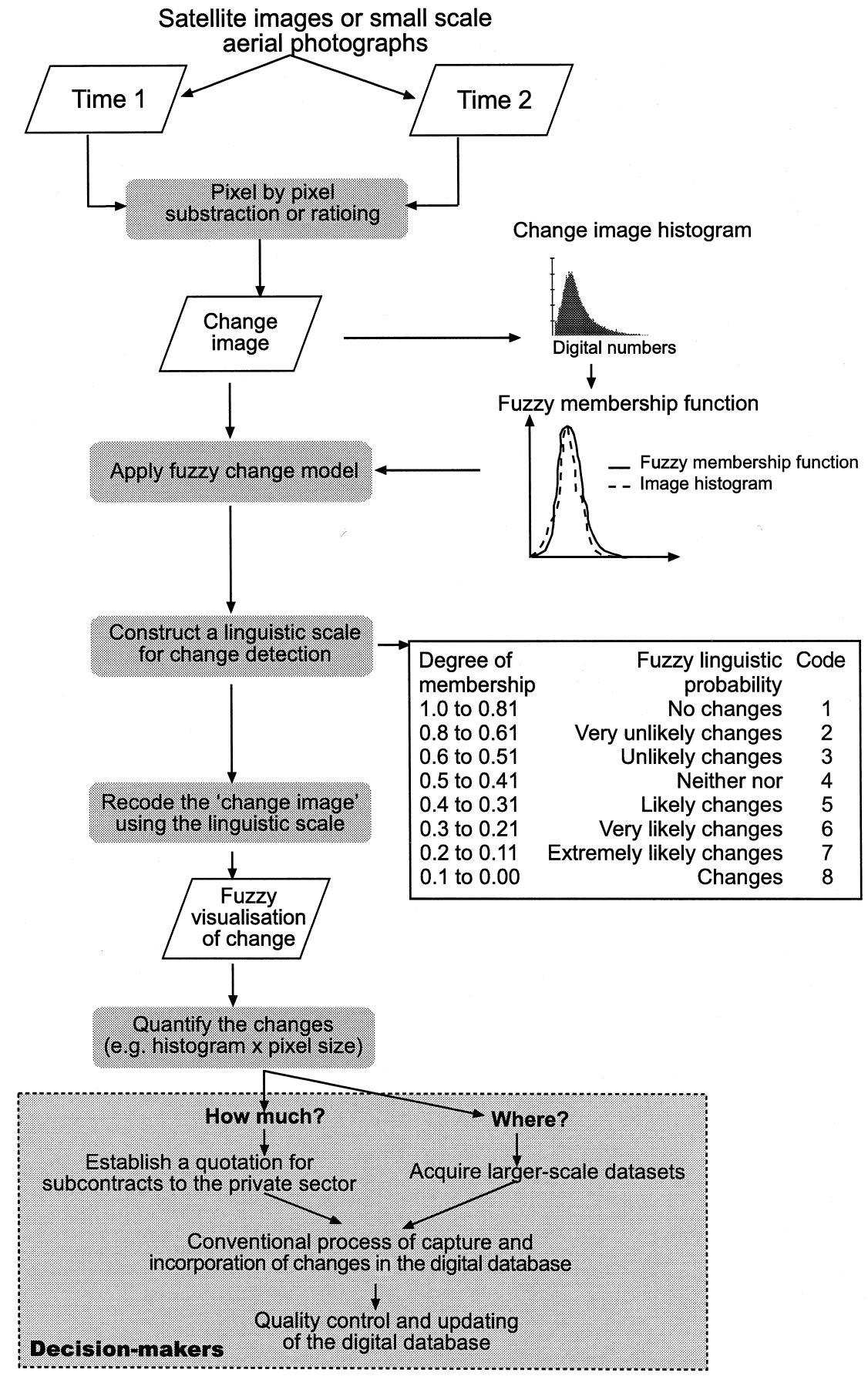

Fig. 3. The methodological approach of change detection using fuzzy sets.

Image difference and image ratioing as described in Jensen (1997) were next performed in both data sets. The change image produced using image differences yielded a Gaussian brightness value distribu- 


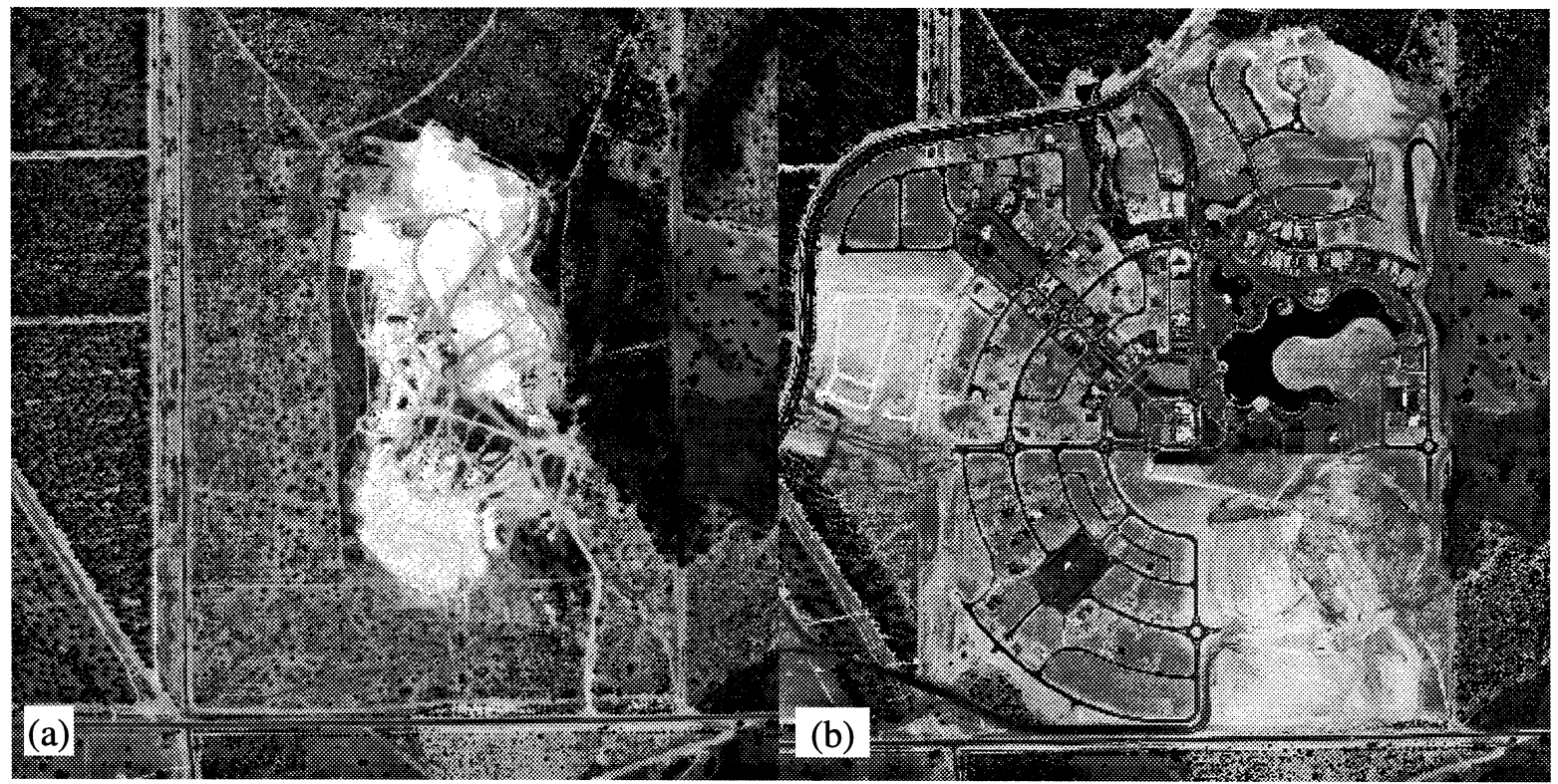

Fig. 4. The Gnangara area. (a) Aerial photograph of January 1992; (b) aerial photograph of January 1996.

tion where pixels of no change are distributed around the mean and pixels representing changes between Time 1 and 2 are found in the tails of the distribution (Fig. 5a). Fig. 5b illustrates the histogram obtained when applying image ratioing. Pixels of no change have a brightness value close to 1 , and pixels of change constitute the tails of the histogram, characterised by a high peak and non-normal distribution.

\subsection{Selecting the membership function}

As indicated in Fig. 3, the next step of the methodology is selecting a membership function that 'fits' to the shape of the change images obtained by ratioing or image difference. To this end, it is assumed that the histogram means represent pixels of no change, with a membership degree of 1 assigned to the fuzzy set 'no change'. Conversely, the tails of the histograms represent pixels of change, thus having a membership of zero in the fuzzy set 'no change'. Applying the concepts presented in Eqs. (2) and (3), the mean corresponds to the standard point of the membership function implemented here, while the tails are the typical points of the function. Table 2 summarises the values of the histograms' mean and tail values corresponding to the change images.

Eqs. (2) and (3) were subsequently applied, modifying the sharpness $(\lambda)$ and inflection $(\nu)$ in order to obtain membership functions of shape coincident with the histograms. Fig. $6 a$ and $b$ show the 'best' membership functions representing a continuous change of the membership degree for the fuzzy set 'no change' from 1 for the mean value of the histograms (depicting areas of no change) decreasing to 0 for the tails of the histograms (indicating changes).

Table 1

General characteristics of the data sets used for the practical study

\begin{tabular}{lllll}
\hline Data set and scale & Date & No. of ground control points & RMSE $(\mathrm{m})$ & Original pixel resolution $(\mathrm{m})$ \\
\hline Aerial photograph, 1:40,000 & 13.01 .92 & 18 & 8.2 & 1 \\
Aerial photograph, 1:20,000 & 08.01 .96 & 11 & 2.9 & 1.24 \\
\hline
\end{tabular}



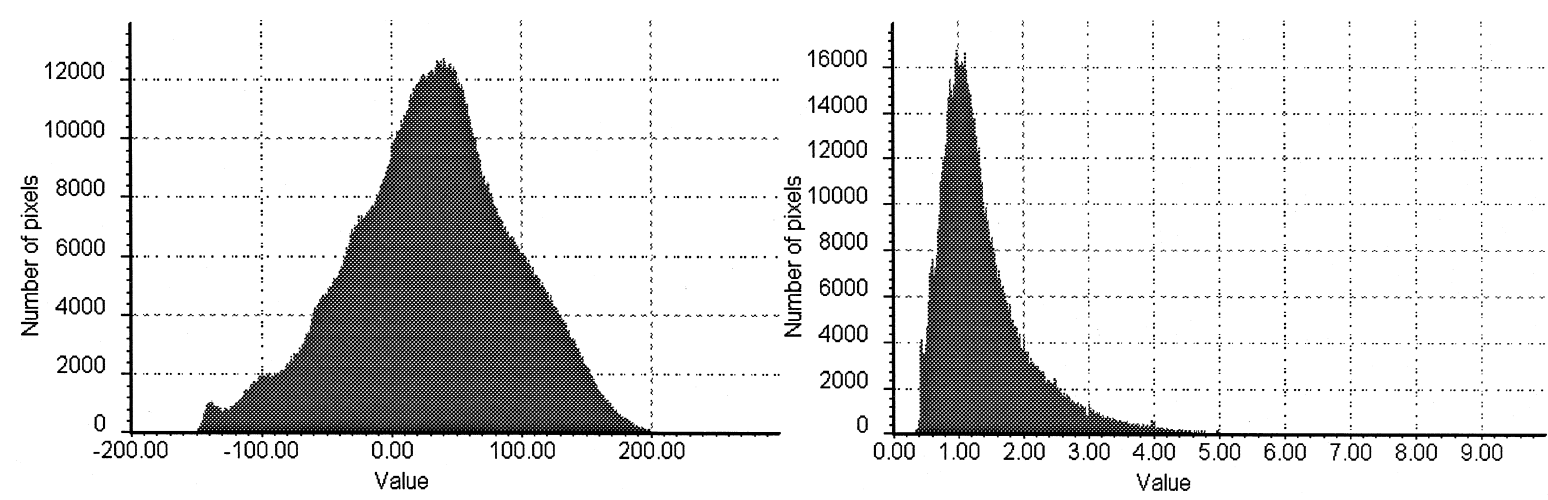

Fig. 5. Histograms of the change image of the Gnangara area (a) using image difference and (b) applying image ratio.

\subsection{Constructing a linguistic scale}

The methodology presented here considers that experts most often use linguistic constructs to describe changes. The experts' knowledge, in the way of terms expressing degrees of likelihood such as the possibility of a change to have occurred, can be ranked and expressed as certainty factors within the range of 0 to 1 . Instead of a continuously measured function, the linguistic scale adopted here uses eight different fuzzy quantifiers to subdivide the fuzzy membership function (see Fig. 3).

\subsection{Generating membership degrees}

The linguistic scale depicted in Fig. 3 represents the degrees of possibilities for change. For instance, all the pixel values with a membership degree of 0.11 to 0.2 represent areas of extremely likely changes. The upper and lower boundaries of the eight 'degrees of likelihood' presented in Fig. 3 were derived from Fig. $6 a$ and $b$ and are presented in Table 3. Subsequently, these values were used to slice the change image into eight classes, as presented in Fig. 7.

\subsection{Fuzzy visualisation of changes}

The final step assigns a grey scale to the sliced change images. To this end, a scale ranging from white to represent pixels with an absolute certainty of 'no change', to black indicating areas where there is absolute certainty that changes have occurred, was designed. In such a way it is easy to visualise not only where changes have occurred, but also how likely these changes were. This concept is better illustrated in Fig. 7.

A statistical analysis to compare the agreement between the surface area mapped for each fuzzy class, when using image ratioing (Fig. 7b) or differencing (Fig. 7a) techniques, was carried out. Histograms of the output images were computed to obtain the area (in percent) assigned to each class. The results reported in Table 4 show that main disagreements between the methods occur at the extremes of the fuzzy change classes, that is, the

Table 2

Values for the sharpness $(\lambda)$ and inflection $(\nu)$ parameters, standard and typical points characterising the membership function of the change images

\begin{tabular}{|c|c|c|c|c|c|c|}
\hline \multirow[t]{2}{*}{ Change image } & \multicolumn{2}{|c|}{ Sharpness } & \multicolumn{2}{|c|}{ Inflection } & \multirow[t]{2}{*}{ Standard point } & \multirow[t]{2}{*}{ Typical points } \\
\hline & $\mathrm{MI}^{\mathrm{a}}$ & $\mathrm{MD}^{\mathrm{b}}$ & $\mathrm{MI}^{\mathrm{a}}$ & $\mathrm{MD}^{\mathrm{b}}$ & & \\
\hline Image Difference & 1.5 & 1.5 & 0.8 & 0.9 & 42 & -164 and 221 \\
\hline
\end{tabular}

\footnotetext{
${ }^{\mathrm{a}} \mathrm{MI}$ : Monotonically increasing part of the function.

${ }^{\mathrm{b}} \mathrm{MD}$ : Monotonically decreasing part of the function.
} 

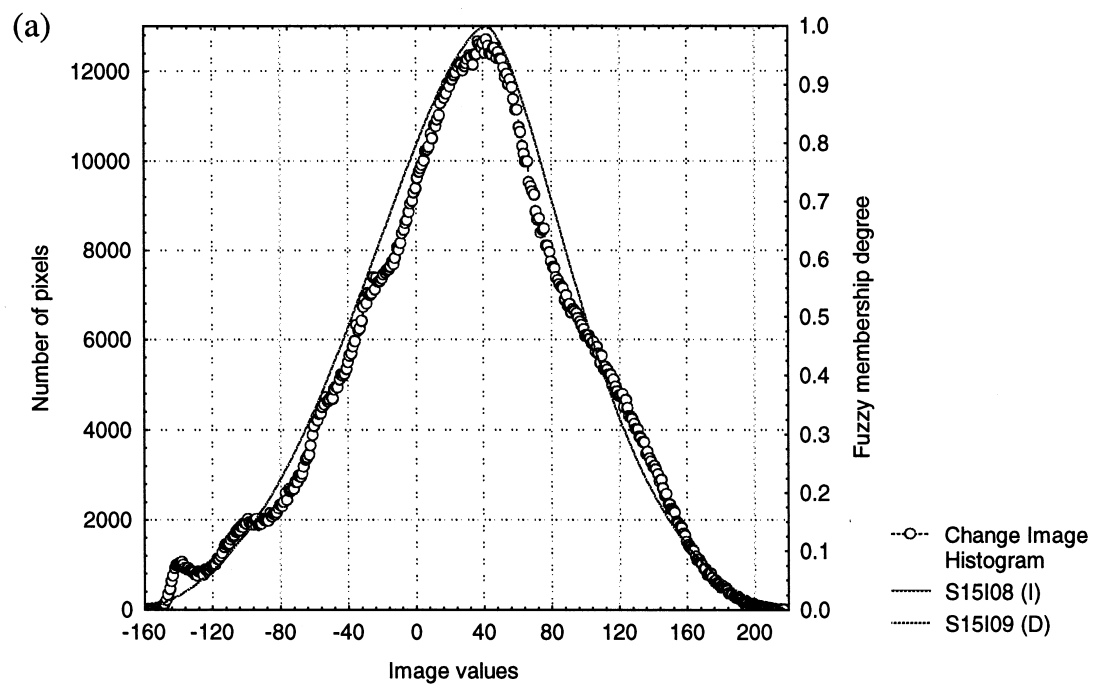

(b)
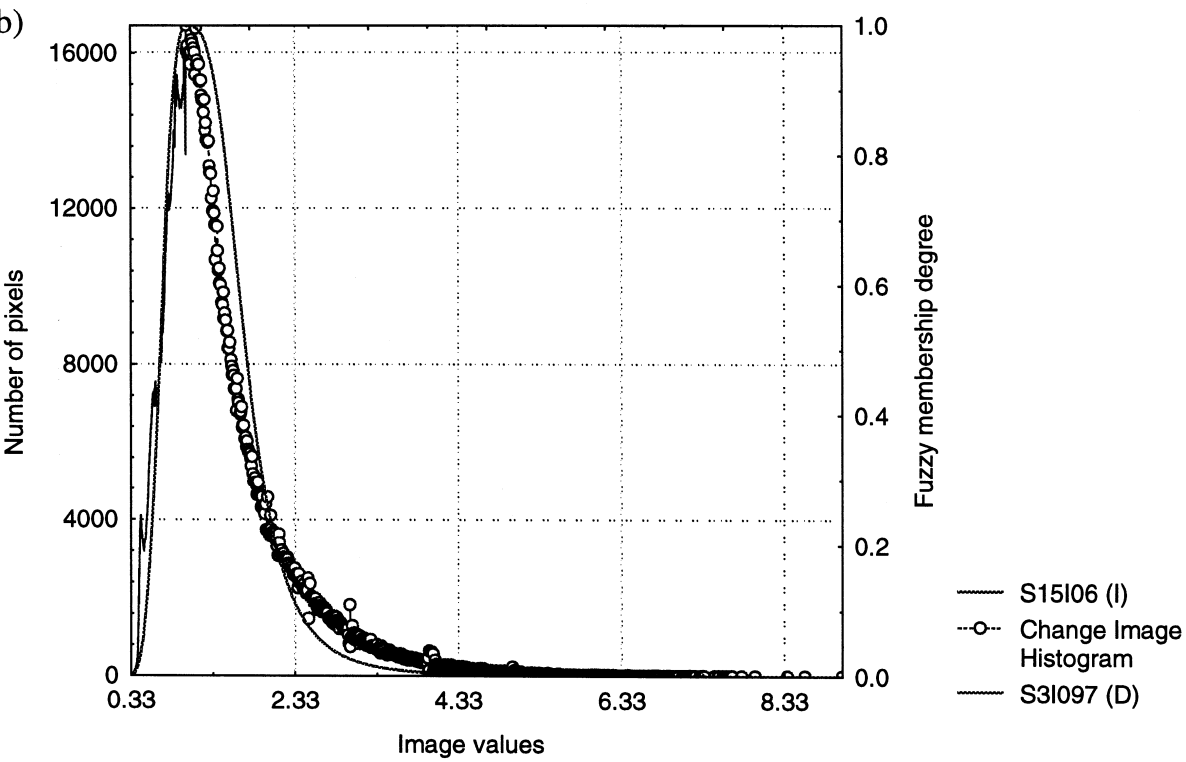

Fig. 6. Best membership function for the Gnangara change image. (a) image difference, (b) image ratio. ( $I)$ and $(D)$ : Parameters corresponding to the increasing and decreasing part of the function, respectively. For instance, S15I08 ( $I$ ) means a sharpness value equal to 1.5 and inflection of 0.8 for the increasing part of the function. Likewise, S15I09 $(D)$ means a sharpness value equal to 1.5 and inflection of 0.8 for the decreasing part of the function.

'change' and 'no change' classes. Further spatial analysis by overlying Fig. 7a and $\mathrm{b}$ in a GIS, indicated that disagreements were in the range of one membership interval. For instance, the high discrepancy reported for the class 'change' (code 8 in Table 4) was mostly related to a mix labelling between codes 7 and 8 . This means that areas labelled as 'extremely likely changes' (code 7) when applying image difference technique, were identified as 'change' (code 8) when ratioing the images. The discrepancies in the spatial extent of 'very unlikely changes' and 'no change' areas (code 2 and 1, 
Table 3

Fuzzy class intervals for the Gnangara data set

\begin{tabular}{|c|c|c|c|c|}
\hline \multirow[t]{2}{*}{ Likelihood code } & \multicolumn{2}{|c|}{ Image difference membership function } & \multicolumn{2}{|c|}{ Image ratio membership function } \\
\hline & Increasing part & Decreasing part & Increasing part & Decreasing part \\
\hline 1 & 2 to 70 & & 0.81 to 1.47 & \\
\hline 2 & -24 to 1 & 71 to 89 & 0.76 to 0.8 & 1.48 to 1.64 \\
\hline 3 & -37 to -25 & 90 to 99 & 0.69 to 0.72 & 1.65 to 1.72 \\
\hline 4 & -50 to -38 & 100 to 110 & 0.66 to 0.68 & 1.73 to 1.81 \\
\hline 5 & -65 to -51 & 111 to 123 & 0.62 to 0.65 & 1.82 to 1.93 \\
\hline 6 & -83 to -66 & 124 to 139 & 0.58 to 0.61 & 1.94 to 2.08 \\
\hline 7 & -107 to -84 & 140 to 161 & 0.52 to 0.57 & 2.09 to 2.35 \\
\hline 8 & -64 to -108 & 162 to 221 & 0.33 to 0.51 & 2.36 to 9.04 \\
\hline
\end{tabular}

respectively) can be justified in the same manner. However, this comparative analysis could not highlight the superiority of one particular technique.
Ground truth data were available to check the reliability of the mapped changes. Accordingly, randomly distributed checkpoints were selected on the

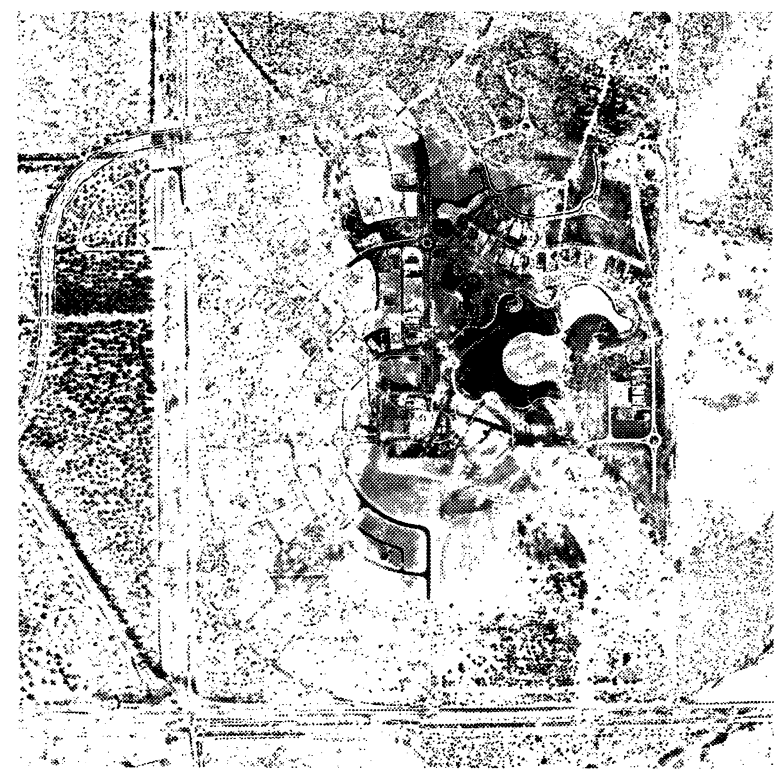

(a) Fuzzy change image derived from image difference

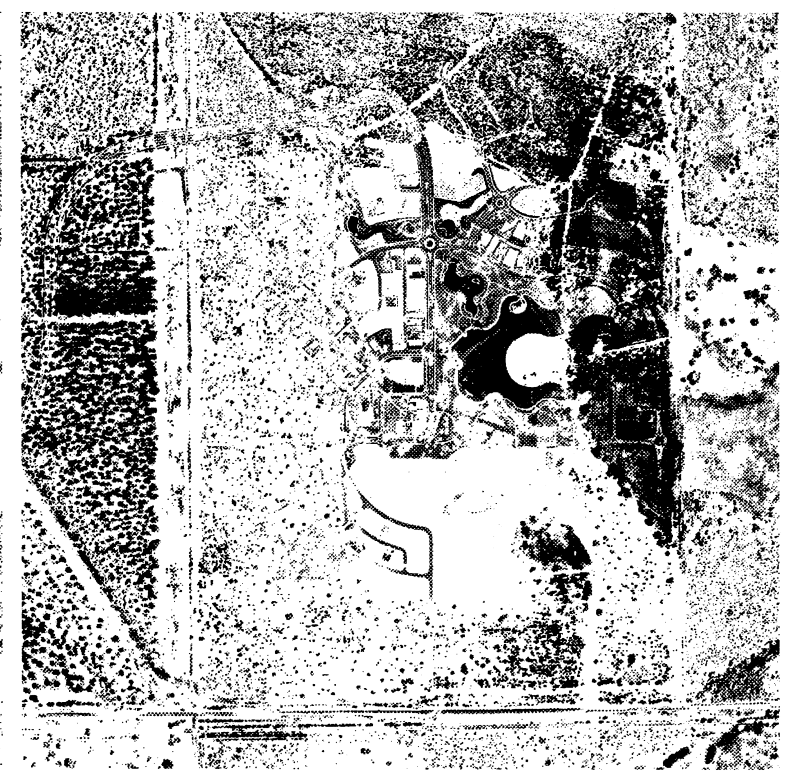

(b) Fuzzy change image derived from image ratio

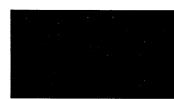

Changes

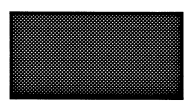

Extremely likely changes

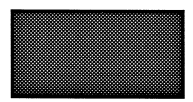

\section{Very likely changes}

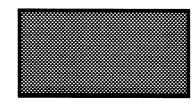

Likely changes

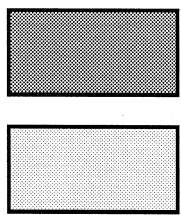

Some likelihood

Unlikely changes to no-changes

Fig. 7. Fuzzy visualisation of the Gnangara change image. (a) Image difference, (b) image ratio. 
Table 4

Analysis of the discrepancies in the extent and certainty of changes as produced by image differencing or ratioing techniques

\begin{tabular}{llllll}
\hline $\begin{array}{l}\text { Likelihood } \\
\text { code }\end{array}$ & Fuzzy linguistic probability & $\begin{array}{l}\text { Image ratio } \\
\text { (percentage identified) }\end{array}$ & $\begin{array}{l}\text { Image difference } \\
\text { (percentage identified) })^{\mathrm{a}}\end{array}$ & $\begin{array}{l}\text { Discrepancy between } \\
\text { techniques }^{\mathrm{b}}\end{array}$ & $\begin{array}{l}\text { Fuzzy } \\
\text { interval }\end{array}$ \\
\hline 1 & No change & 50.70 & 43.16 & 7.53 & $0.81-1.00$ \\
2 & Very unlikely change & 12.32 & 19.68 & 7.37 & $0.61-0.80$ \\
3 & Unlikely change & 4.75 & 8.27 & 3.52 & $0.51-0.60$ \\
4 & Neither likely nor unlikely & 4.06 & 7.20 & 3.13 & $0.41-0.50$ \\
5 & Likely change & 4.71 & 6.96 & 2.25 & $0.31-0.40$ \\
6 & Very likely change & 4.74 & 6.02 & 1.28 & $0.21-0.30$ \\
7 & Extremely likely change & 6.06 & 5.33 & 0.73 & $0.11-0.20$ \\
8 & Change & 12.67 & 3.39 & 9.28 & $0.00-0.10$ \\
\hline
\end{tabular}

${ }^{\text {a }}$ Percentage labelled as, e.g., 'change areas', relative to the total surface covered in the study.

${ }^{\mathrm{b}}$ Expressed in percent.

change image and weighed against the ground data. Although the procedure relied on visual assessment, a good agreement between 'real' and 'mapped' changes was observed. As mentioned at the end of this paper, better methods for accuracy assessment, accounting for not only the change/no change agreements but also the fitness to the linguistic scale, shall be adopted in the future.

\section{Applications of the fuzzy change model to map revision}

\subsection{Issues considered during the map revision pro- cess}

The output of the fuzzy change model can be used by managers and decision-makers of topographic organisations to address the following issues: (i) When do I need to undertake map revisions tasks assuming there is not a fix revision cycle scheme? (ii) Where have spatial changes occurred? (iii) How much change has occurred in an area? (iv) Does the magnitude of changes justify the expenditure of resources for map revision? (For example, the organisation could work out a threshold for decision-making based on cost-effectiveness or users' demands.) (v) If the map revision tasks are undertaken, what is the cost estimation for sub-contracts to the private sector?

As proposed here, topographic organisations can derive 'working rules' for cost-effective decisions based on the magnitude of changes that have occurred within an area. For example, rules such as 'IF more than $20 \%$ of the area reports likely to absolutely likely changes, THEN proceed with map revi-

Table 5

Estimation of the areal extent and certainty of changes applying the image difference technique

\begin{tabular}{llllll}
\hline Number of pixels & Area $(\mathrm{ha})^{\mathrm{a}}$ & Percentage $^{\mathrm{b}}$ & Likelihood code & Fuzzy linguistic probability $^{\text {Fuzzy interval }}$ \\
\hline 788,928 & 78.9 & 43.2 & 1 & No change & $0.81-1.0$ \\
359,778 & 36.0 & 19.7 & 2 & Very unlikely change & $0.61-0.8$ \\
151.164 & 15.1 & 8.3 & Unlikely change & $0.51-0.6$ \\
131,535 & 13.2 & 7.2 & 4 & Neither likely nor unlikely & $0.41-0.5$ \\
127,168 & 12.7 & 6.9 & 5 & Likely change & $0.31-0.4$ \\
109,992 & 11.0 & 6.0 & 6 & Very likely change & $0.21-0.3$ \\
97,387 & 9.7 & 5.3 & 7 & Extremely likely change & $0.11-0.2$ \\
61,952 & 6.2 & 3.4 & 8 & Change & $0.00-0.1$ \\
\hline
\end{tabular}

${ }^{\mathrm{a}}$ Area $(\mathrm{ha})=0.0001$ ha $\times$ number of pixels, as the data set has a $1-\mathrm{m}$ pixel resolution.

${ }^{\mathrm{b}}$ Percentage $=($ Area of a likelihood code $/$ Total area of the change image $) \times 100$. 
sion and updating tasks', can be implemented capitalising on the experts' knowledge about the area being considered.

Multiplying the surface covered by a pixel by the number of pixels falling within a specific range and dividing by the total area provides the percentage of predicted changes, ranked according to their certainty. For instance, the results presented in Table 5 imply that $14.7 \%$ of the area is ranked as very likely to absolute likely to have changed, $42.1 \%$ of the area is labelled as likely to very unlikely to have changed and in $43.2 \%$ of the area there is an absolute certainty that no changes have occurred within the period of time considered.

\section{Conclusions}

The proposed method presents some improvements in the field of change detection and visualisation of the certainty and magnitude of changes. In addition, a system to prioritise areas targeted for map and database revision based on a manager's criteria of a cost-effective threshold of change is presented. It should be noted that the method accounts only for spatial changes of an object or surface feature. Changes to the attributes (e.g., from residential to commercial use) are not likely to be detected, unless significant spatial changes occur.

Furthermore, the accuracy of the method is dependent on the spatial resolution of the remotely sensed data used, conditioning the detectability of point, line and area features. Authors like Konecny (1990) and Albertz and Tauch (1994) have evaluated the resolving power of various satellite and airborne sensors and the minimum size of point, line and area features that can be detected, thus determining their adequacy for detecting changes relevant for map revision at particular map scales.

As mentioned above, there are many issues and questions that remain to be addressed, mainly related to accuracy assessment of the change detection approach presented here. Further research is currently being undertaken to assess the accuracy of using evaluation techniques such as Kappa analysis, in contrast to the fuzzy approaches proposed by Gopal and Woodcock (1994). In addition, a method for evaluating the correspondence of the change image with the fuzzy linguistic scale should be designed.

The theory and methods for change detection assessment using fuzzy sets as discussed in this paper constitute a solution for identifying change and objectively defining thresholds for separating areas of relative change/no-change in the change image. It is anticipated that the approach can be applied to other fields, such as natural resource survey, estimation and monitoring of deforested areas and detecting changes in the condition of agricultural crops. Conclusions on the methodology can be summarised as follows.

- The method provides an alternative solution to the estimation of spatial changes solely by using visual interpretation.

- The approach can be used jointly with current map revision cycles. For organisations such as the Department of Administration of Western Australia, charged with the update of a digital database with approximately 1960 map tiles or the Ordnance Survey, UK with 229,000 topographic sheets (Ridley et al., 1997), it is helpful to identify the areal extent and location of the areas undergoing a large number of changes. In this way, a 'sub-program' of prioritisation of map updating within a specific map revision cycle scheme can be established.

- The method is adequate for multi-scale approaches. For instance, Landsat TM satellite images can be obtained for an area requiring revision. The fuzzy change model would then show the general extent and location of the changes so that higher resolution datasets could be acquired for detailed change detection in critical areas.

\section{Acknowledgements}

The author would like to thank Messrs. Peter Eddie, Ron Wyllie and Ray Webster of the Geographic Data Service at the Department of Land Administration of Western Australia (DOLA) for their valuable discussions about the problems related to map revision and updating tasks in extensive areas. Special thanks are also extended to DOLA for the provision of the dataset used in this research, $\mathrm{Mr}$. Dan Sandison for the geometric correction of the data set and to the anonymous reviewers that provided critical comments to improve the paper. 


\section{References}

Albertz, J., Tauch, R., 1994. Mapping from space: cartographic applications of satellite image data. GeoJournal 32 (1), 29-37.

Burrough, P.A., 1989. Fuzzy mathematical methods for soil survey and land evaluation. Journal of Soil Science 40, 477-492.

Dombi, J., 1990. Membership function as an evaluation. Fuzzy Sets and Systems 35, 1-21.

Gopal, S., Woodcock, C., 1994. Theory and methods for accuracy assessment of thematic maps using fuzzy sets. Photogrammetric Engineering and Remote Sensing 60 (2), 181-188.

Jensen, J., 1996. Introductory Digital Image Processing: A Remote Sensing Perspective, 2nd edn. Prentice-Hall, Englewood Cliffs, NJ, 330 pp.

Jensen, J., 1997. Principles of change detection using digital remote sensor data. In: Star, Estes, McGwire (Eds.), Integration of Geographic Information Systems and Remote Sensing. Cambridge Univ. Press, pp. 37-54.

Jensen, J., Toll, D., 1982. Detecting residential land-use development at the urban fringe. Photogrammetric Engineering and Remote Sensing 48 (4), 629-643.

Konecny, G., 1990. Review of the latest technology in satellite mapping. Interim Report, Intercommission Working Group I/IV on International Mapping and Remote Sensing Satellite Systems of ISPRS. Hannover, Germany, 14, pp. 11-21.

McBratney, A., de Gruijter, J., 1992. A continuum approach to soil classification by modified fuzzy $k$-means with extragrades. Journal of Soil Science 43, 159-175.

McBratney, A., Moore, A., 1985. Application of fuzzy sets to climate classifications. Agricultural and Forest Meteorology $35,165-185$.

Metternicht, G., Lodwick, G., Sandison, D., Gonzalez, S., Wright, G., 1997. Digital map revision and topographic database updating: a feasibility study. Report produced for the Department of Land Administration of Western Australia, Perth, WA, 50 pp.

Newby, P., 1996. Working group IV/3 report and review of progress in map and database revision. Int. Arch. Photog. Rem. Sens. 31 (B4), 598-603.

Parra, G., Mouchot, M., Roux, C., 1996. A multitemporal land cover change analysis tool using change vector and principal component analysis. Proc. IGARSS'96 Symp. 1, 1753-1755.

Ridley, H., Atkinson, P., Aplin, P., Muller, J., Dowman, I., 1997. Evaluating the potential of the forthcoming commercial US high-resolution satellite sensor imagery at the Ordnance Survey. Photogrammetric Engineering and Remote Sensing 63 (8), 997-1005.

Robinson, V.B., 1988. Some implications of fuzzy set theory applied to geographic databases. Computers, Environment and Urban Systems 12, 89-97.

Singh, A., 1989. Digital change detection techniques using remotely sensed data. International Journal of Remote Sensing 10 (6), 989-1003.

Wang, F., 1990. Improving remote sensing image analysis through fuzzy information representation. Photogrammetric Engineering and Remote Sensing 56 (8), 1163-1169.

Zadeh, L., 1965. Fuzzy sets. Information and Control 8, 338-353.

Zadeh, L., 1984. Making computers think like people. IEEE Spectrum 8, 26-32. 